\title{
Drug Induced Interstitial Lung Disease
}

\author{
Martin Schwaiblmair ${ }^{*}$, , Werner Behr ${ }^{2}$, Thomas Haeckel ${ }^{3}$, \\ Bruno Märkl ${ }^{4}$, Wolfgang Foerg ${ }^{5}$ and Thomas Berghaus ${ }^{1}$
}

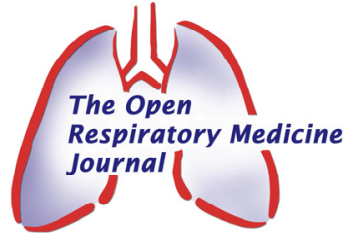

\author{
${ }^{I}$ Department of Internal Medicine I, ${ }^{2}$ Department of Laboratory Medicine, ${ }^{3}$ Department of Diagnostic Radiology, \\ ${ }^{4}$ Department of Pathology, ${ }^{5}$ Department of Clinical Pharmacy, Klinikum Augsburg, Ludwig-Maximilians-University of \\ Munich, Germany
}

\begin{abstract}
With an increasing number of therapeutic drugs, the list of drugs that is responsible for severe pulmonary disease also grows. Many drugs have been associated with pulmonary complications of various types, including interstitial inflammation and fibrosis, bronchospasm, pulmonary edema, and pleural effusions. Drug-induced interstitial lung disease (DILD) can be caused by chemotherapeutic agents, antibiotics, antiarrhythmic drugs, and immunosuppressive agents. There are no distinct physiologic, radiographic or pathologic patterns of DILD, and the diagnosis is usually made when a patient with interstitial lung disease (ILD) is exposed to a medication known to result in lung disease. Other causes of ILD must be excluded. Treatment is avoidance of further exposure and systemic corticosteroids in patients with progressive or disabling disease.
\end{abstract}

Keywords: Lung, adverse drug reaction, drug-induced lung disease, mechanism of pulmonary toxicity, diagnosis, treatment, review.

\section{INTRODUCTION}

The lungs are a target for a variety of possible toxic substances because of their large contact surface. They can also act as a metabolism site for certain substances. Drugs can induce specific respiratory reactions or the lungs may be affected as part of a generalized response. More than 380 medications are known to cause drug-induced respiratory diseases, the true frequency is unknown $[1,2]$. The number of drugs, that cause lung disease, will undoubtedly continue to increase as new agents are developed.

To minimize the potential morbidity and mortality from drug-induced respiratory diseases, all health care providers should be familiar with the possible adverse effects of the medications they prescribe. The person-to-person variability of a drug response is a major problem in clinical practice and in drug development. The variability in drug response among patients is multifactorial, including extrinsic factors like environmental aspects and also genetic and intrinsic factors that affect the disposition of a certain drug.

Drug-induced lung injury may involve the airways, lung parenchyma, mediastinum, pleura, pulmonary vasculature, and/or the neuromuscular system. The most common form of drug-induced lung toxicity is drug-induced interstitial lung disease (DILD). Oral and parenteral routes of drug administration are most frequently cited as causing DILD; however, nebulized and intrathecal administration have been also been implicated. Pulmonary drug toxicity may result from a direct or indirect drug effect. Direct effects may be

*Address correspondence to this author at the Department of Internal Medicine I, Klinikum Augsburg, University of Munich, Stenglinstr. 2, D86156 Augsburg, Germany; Tel: (0049)-821-400-2849; Fax: (0049)-821400-2966; E-mails: martin.schwaiblmair@klinikum-augsburg.de either idiosyncratic or due to a toxic reaction of the drug or one of its metabolites.

Recognition of drug-induced lung disease, however, is difficult because the clinical, radiological, and histological findings are nonspecific. The connection with drug use and the development of related inflammatory damage or idiosyncratic toxicities is hard to recognize and objectify, especially in those cases using multiple drugs [3].

\section{EPIDEMIOLOGY}

Many drugs are effective in only 25 to 60 percent of patients, and more than 2 million cases of adverse drug reactions occur annually in the United States, including 100.000 deaths [4-6]. Hitchen et al. reported that adverse drug reactions (ADR) are the cause of 250.000 hospitalizations per year in the United Kingdom [7]. A Swedish study has implicated adverse drug reactions as $7^{\text {th }}$ most common cause of death [8]. A large prospective study of adverse drug reaction in UK hospital in-patients suggests that at least 1 in 7 in-patient episodes is complicated by an adverse drug reaction and the incidence of $14.7 \%$ is comparable with previous studies [9]. In UK, $0.9 \%$ of total hospital admissions represent adverse drug reactionassociated admissions [10]. Over the period between 1999 and 2008 the annual number of adverse drug reactions increased by $76.8 \%$, and in-hospital mortality rate increased by $10 \%$. An estimated calculation in the Netherlands showed that about 430 million euros could be saved each year when side effects are reduced [6]. In the United States, an estimated $0.3 \%$ of hospital deaths are drug related [5]. Up to $10 \%$ of patients who receive chemotherapeutic agents develop an ADR in their lungs [11]. Exact frequency of drug-induced pulmonary toxicity is unknown. Several studies suggest that drug-induced pulmonary toxicity is underdiagnosed worldwide. Global incidence of interstitial 
lung disease (ILD) is not clearly known but $2.5-3 \%$ of cases are drug induced $[12,13]$. The list and frequency of toxicity of all drugs implicated in lung injury can be found elsewhere [14].

\section{RISK FACTORS FOR DILD}

The likelihood of developing adverse pulmonary effects secondary to drugs remains largely unpredictable and idiosyncratic. Patients likely to develop DILD are those receiving chemotherapy, those with inflammatory conditions such as rheumatoid arthritis and inflammatory bowel disease, and those receiving concurrent multiple toxic agents. Some of the known risk factors are as follows:

\section{Age}

In general, both extremes of age (i.e. childhood and old age) are associated with an increased risk of drug toxicity. The elderly patient is more prone to develop severe side effects, partly because of the fact that the excretory function of the kidney is lower, the liver blood perfusion is diminished and the overall metabolic function is changed. For instance, Simpson et al. [15] showed in a retrospective review of bleomycin pulmonary toxicity, that the fatal cases of pulmonary toxicity were older than the remaining patients and for patients aged over 40 years, especially those with renal function in the lower range of normal, the risk of developing fatal toxicity may exceed $10 \%$.

\section{Sex}

In long-term nitrofurantoin prophylaxis, a chronic form of pulmonary reactions has been reported to occur after 8 months to 16 years of treatment [16]. Most of those affected have been elderly and most have been females. However at yet, there is no scientific evidence in the literature that the gender influences the risk of DILD.

\section{Ethnicity}

There may be racial differences in the incidence of DILD. In Japan, cases of lung diseases presumably induced by gefitinib have provided important findings, including evidence about racial differences in the DILD and pathological variety of the lung diseases [17]. However, it is difficult to determine the relative incidence of DILD during the drug-development phase, because the events are too rare and the sample sizes are too small to make reliable estimates from the results of clinical studies. Acute and organizing diffuse alveolar damage associated with Bortezomib were also first reported in Japan and subsequently in African American patients with severe pneumonitis $[18,19]$. Ethnic influences could be also observed by leflunomide and tacrolimus [20, 21]. Genetic polymorphisms may help explain why some groups of patients have the expected response to pharmacotherapy whereas others experience toxicity or therapeutic failure [22-26].

\section{Dose}

In several cases, such as therapies with amiodarone, bleomycin or BCNU, the dose has been found to be a risk factor for a drug to cause ILD [1, 27-31]. Although this affirmation is not universal, physicians should keep in mind this possibility and monitor a drug dose according to the published information.

\section{Oxygen}

Lung tissue is vulnerable to the toxic effects of oxygen and oxygen damage readily occurs. The lungs are normally equipped with an extensive antioxidant network to protect against tissue damage by reactive oxygen species. This network may be insufficient and this situation of inadequate protection is called oxidative stress. It is striking that numerous conditions that lead to ILD may cause reactive oxygen species and result in oxidative stress [32]. Disruption of the oxidant/antioxidant balance is important in the pathogenesis of acute lung injury and acute respiratory distress syndrome (ARDS) [33]. Amiodarone is damaging to the lungs via transfer of electrons to $\mathrm{O} 2$.

\section{Drug Interaction}

The role of drugs taken concomitantly may be important. Drugs in the same therapeutic class can often induce similar pulmonary toxicity pattern [34]. Hazardous associations have been reported with the coadministration of cisplatin and bleomycin, which can increase the risk of bleomycininduced interstitial lung disease. The combination therapy of gemcitabine and bleomycin is very toxic too.

\section{Radiation}

DNA damage and repair proteins are involved in many types of lung injury and repair. Radiation can be injure the lung by this mechanisms and radiation therapy in combination with chemotherapy may be synergistic [1]; for instance, conjoint radiation therapy is associated with high rates of lung toxicity by bleomycin. Interstitial pneumonitis after fractionated total body irradiation in preparation for allogenic bone marrow transplantation occurred with a significant incidence of $20 \%$ [35].

\section{Underlying Lung Disease}

Preexisting lung disease as an important risk factor is controversial. A large-scale prospective case-control study of risk factors of acute lung diseases/interstitial pneumonia was performed in a cohort of patients with non small lung cancer who had or had not been treated with gefitinib [17]. By logistic regression analysis, the following risk factors were identified to be most commonly associated with a poor prognosis: age $\geq 65$ years, smoking habit, reduced lung function and pre-existing interstitial pneumonia. The hitherto existing literature states the prevalence of methotrexate pneumonitis to be 3.5-7.6\% [36-38]. Sathi $N$ et al. designed the largest ongoing prospective study and the results suggest that methotrexate pneumonitis does not occur as often as previously thought with an incidence of one case every 192 patient years [39]. Furthermore, ILD is reported in $1 \%$ of those taking leflunomide and $0.6 \%$ of patients receiving etanercept $[40,41]$. The possible link between leflunomide and ILD has evoked increasing concern. $1.2 \%$ in Japanese patients with rheumatoid arthritis who received leflunomide developed de novo or exacerbated ILD; and pre-existing ILD was the most important risk factor for leflunomide-induced ILD [42]. On the other hand, Suissa et al. [20] conducted a population-based epidemiologic study and concluded that patients treated with leflunomide who have no history of methotrexate use and no existing ILD carry no excess risk of ILD. The increase in the risk of ILD associated with leflunomide is restricted to the subgroup of patients with a 
history of methotrexate use or existing ILD (relative risk 2.6), and this appears to be the result of channeling bias.

\section{CAUSES OF DRUG-INDUCED INTERSTITIAL LUNG DISEASE}

The major representatives of DILD include cytotoxic, cardiovascular, anti-inflammatory, antimicrobial, biological agents and miscellaneous drugs (Table 1):

Table 1. Drugs Associated to the Development of Interstitial Lung Disease

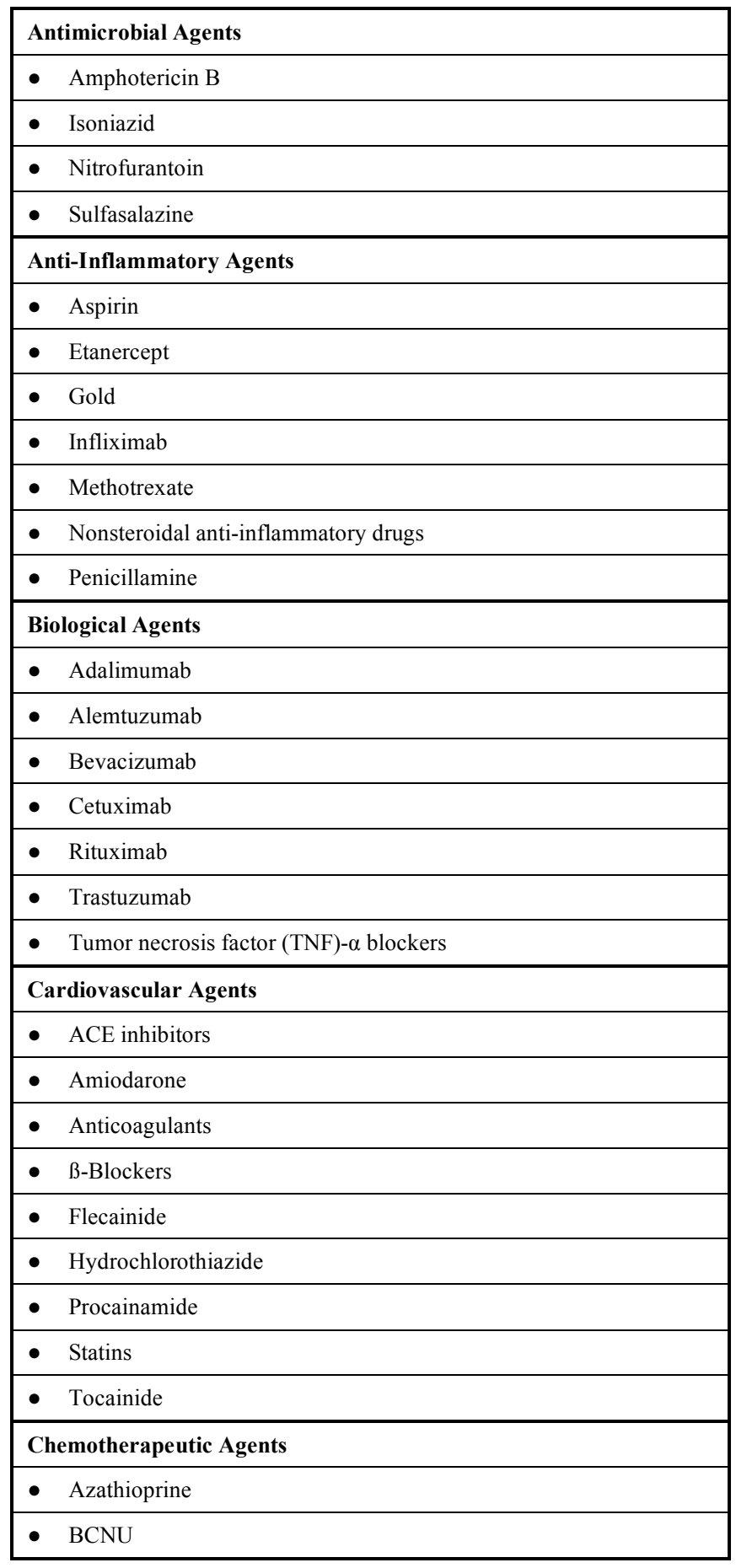

(Table 1) contd......

Chemotherapeutic Agents

- Bleomycin

- Bortezomib

- Busulfan

- Carmustine

- Chlorambucil

- Colony-stimulating factors

- Cyclophosphamide

- Cytarabine

- Deferoxamine

- Docetaxel

- Doxorubicin

- Erlotinib

- Etoposide

- Fludarabine

- Flutamide

- Gefitinib

- Gemcitabine

- Hydroxyurea

- Imatinib

- Interferons

- Lomustine

- Melphalan

- Methotrexate

- Methyl-CCNU

- Mitomycin-C

- Nitrosoureas

- Paclitaxel

- Procarbazine

- Thalidomide

- Vinblastine

- Zinostatin

Miscellanous

- Bromocriptine

- Carbamazepine

- Cabergolide

- Methysergide

- Penicillamine

- Phenytoin

- Sirolimus

- Talc 


\section{Cytotoxic Drugs}

Any chemotherapeutic drug can adversely affect the lung, but the drugs most commonly implicated in lung toxicity are bleomycin, carmustine, busulfan, and cyclophosphamide [43-46]. Approximately 1-10\% of patients taking one of these drugs are affected. Bleomycin is the drug most commonly studied as a cause of DILD. Larger studies have shown that rates of $8-10 \%$ with some degree of lung injury have been observed [47]. Symptoms may develop earlier than 4 weeks and later than 10 weeks following chemotherapy and the damage is predominantly at the lung base. Busulfan toxicity causes drug-induced pulmonary damage after prolonged exposure, usually after 34 years of therapy [48]. Cyclophosphamide causes earlyonset ILD with a low incidence, estimated at less than $1 \%$, but cyclophosphamide may also cause later damage [48].

\section{Cardiovascular Agents}

Amiodarone is the most common drug related to cardiovascular pulmonary abnormalities. It affects as many as $6 \%$ of individuals receiving the drug and, amongst these cases, fatality rates range from $10-20 \%$ [49]. Statin-induced DILD has been reported with most statins, suggesting that statin-induced ILD is a class effect and not a specific statin effect [50].

\section{Anti-Inflammatory Drugs}

Aspirin is the most common anti-inflammatory drug associated with ADR. An ARDS-type syndrome has been described with salicylate toxicity [51]. Methotrexate is increasingly used as an anti-inflammatory agent to treat many disorders. Pulmonary reaction to methotrexate is commonly subacute, with a hypersensitivity-like manifestation [52]. Important drugs with potential DILD are gold, penicillamine, azathioprine and nonsteroidal antiinflammatory drugs (NSAIDs) [53]. NSAIDs may cause acute pulmonary hypersensitivity reactions resulting in bilateral interstitial infiltration and eosinophilic pneumonia. Onset of such a reaction can occur within the first week and up to three years after first exposure [51].

\section{Antimicrobial Agents}

Nitrofurantoin, amphotericin B, sulfonamides, and sulfasalazine are known to cause DILD. For example, nitrofurantoin is associated with acute or chronic pulmonary injury. The acute manifestation of this process is the most common and is thought to be due to a hypersensitivity reaction from the drug. The chronic form includes pulmonary fibrosis and BOOP [54].

\section{Biological Agents}

The development and production of biological agents has grown rapidly over the last 10 years and represented $22 \%$ of all new chemical entities approved for use by United States or European Union regulatory authorities $[55,56]$. Classes of biological agents with reported DILD include tumor necrosis factor (TNF)- $\alpha$ blockers, anti-CD20 antibodies, recombinant interferon (INF) alpha, T-cell antiproliferative agents, or others like cetuximab, bevacizumab, alemtuzumab or trastuzumab [57-59].

\section{Miscellaneous Drugs}

Bromocriptine has been reported to cause pulmonary fibrosis and pleural disease [60]. Belmonte et al. reported of pleuropulmonary toxicity of cabergoline, an ergoline derivate in the treatment of Parkinson`s disease [61].

\section{MECHANISMS IN PULMONARY TOXICOLOGY}

The mechanism of DILD is not fully understood. Pulmonary toxicology can be divided in two broad categories based on the route of exposure to the offending agent. Alveolar and bronchial epithelial cells may be injured by inhalation of a drug or through the vasculature system [13, 62-64]. Various reasons may be put forward to explain why certain drugs cause toxicity specifically in the lungs: (1) Some substances reach higher cell or tissue concentrations in the lung than in other organs. (2) A specific pattern or extent of bioactivation occurs in the lung. (3) The consequences of bioactivation are lung-specific. Furthermore, some foreign compounds may accumulate preferentially in lung tissue [49].

Lung injury that is induced by pneumotoxic agents gives rise to alveolitis and edema. In response to injury to the lung parenchyma, there is an immediate requirement to initiate tissue repair and restore barrier function. Acute injury may progress to chronic inflammation and eventually lead to fibrotic change that ultimately interferes with gas exchange. Chemotherapeutic drugs can additionally cause a direct toxic reaction, and direct toxicity usually occurs over time before manifesting clinically [65-69]. -induced pulmonary fibrosis [69].

Most chemicals do not cause cell toxicity directly; usually some form of biotransformation is required for chemical agents to cause cell injury. This biotransformation process may increase the toxicity of chemicals by producing reactive metabolites. If these reactive metabolites are not readily removed by enzymatic or nonenzymatic reactions, they may cause cell injury and death $[67,70]$.

The concept that some drugs may cause oxidative stress and that their toxicity may be enhanced by the (therapeutic) administration of oxygen is of paramount importance in clinical settings [71]. A disturbance of oxidant/antioxidant system homeostasis may occur. The resulting toxic oxygen species depletes the reducing equivalents and generates oxidative stress [16, 72-74].

Furthermore, we know two other pathophysiologic principles in pulmonary toxicity [75]. First, drugs can act as potential antigens, or haptens, inducing an immune cascade that can lead to immune-mediated lung toxicity. Hypersensitivity reactions do not show a simple doseresponse relationship and require prior sensibilisation to the drug. The immune damage to the lung may be due to drugspecific antibodies or, more likely, drug-specific $\mathrm{T}$ cells. Deposition of antigen-antibody complexes may trigger an inflammatory response, leading to pulmonary edema and interstitial lung disease. Drug-induced systemic lupus erythematodes is an example of immune-mediated lung damage. Secondly, similar to other amphiphilic compounds, some drugs can cause a deposition of phospholipids within cells. For example, amiodarone has been demonstrated to 
produce phospholipidosis in alveolar macrophages and in type 2 cells.

\section{HISTOLOGIC FINDINGS}

As the name implies, the histological abnormalities that characterize DILD generally involve the pulmonary interstitium to a greater extent than the alveolar spaces or airways. Generally when the interstitium acts to any drug injury, the lung must respond to the damage and repair itself. If the exposure persists or if the repair process is imperfect, the lung may be permanently damaged, with increased interstitial tissue replacing the normal capillaries, alveoli, and intact interstitium. Histological changes for most drug reactions are nonspecific, but a limited number of drugs (e.g. amiodarone) produce a characteristic histopathological pattern of involvement, which enables almost instant recognition of the drug etiology (Table 2). For instance, methotrexate causes an acute granulomatous ILD which mimics an opportunistic infection [73].

Table 2. Histopathologic Manifestations of Pulmonary Reactions to Noncytotoxic Drugs

\begin{tabular}{|l|}
\hline Interstitial pneumonia \\
\hline Hypersensitivity pneumonia \\
\hline Bronchiolitis obliterans organizing pneumonia \\
\hline Granulomatous pneumonitis \\
\hline
\end{tabular}

Drugs can produce virtually all histopathological patterns of interstitial pneumonia, including hypersensitivity pneumonitis (HP), organizing pneumonia (OP), diffuse alveolar damage (DAD) and nonspecific interstitial pneumonia (NSIP), eosinophilic pneumonia, bronchiolitis obliterans organizing pneumonia (BOOP), pulmonary hemorrhage, and granulomatous pneumonitis [3]. Most drugs of a comparable class may induce a similar pattern of pulmonary involvement, suggesting a common cytopathic mechanism. But some drugs can produce more than one pattern of histopathological involvement in the same patient. These reactions can manifest acutely, subacutely, or chronically (Table 2 and Fig. 1).

\section{Hypersensitivity Pneumonitis (HP)}

Drug hypersensitivity results from interactions between a pharmacologic agent and the human immune system. Histological the signs of interstitial pneumonia are found with infiltration of the interstitial space by lymphocytes and plasma cells. At this point other interstitial pneumonias have to be considered. Another important feature of HP is the occurrence of small interstitial non-caseating granulomas with variable numbers of giant cells, for example causing by anti-TNF agents, methotrexate and BCG therapy (Table 3 and Fig. 1a).

\section{Organizing Pneumonia}

The characteristic histological finding of $\mathrm{OP}$ is the occurrence of intra alveolar fibroblastic proliferations with production of immature collagen. The lung architecture is typically preserved, and lymphocytes, plasma cells, and histiocytes are present to a variable degree within the (a)

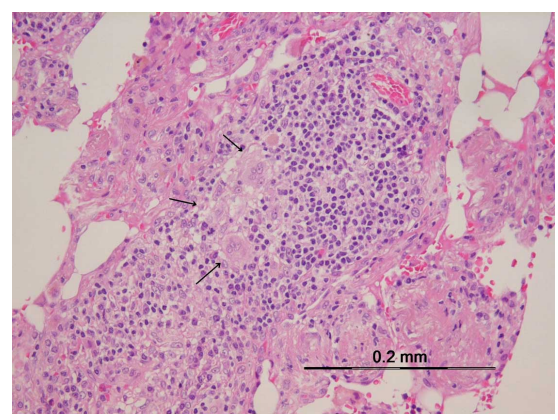

(b)

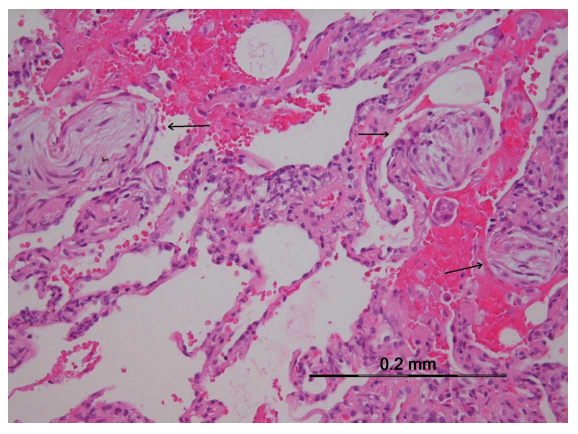

(c)

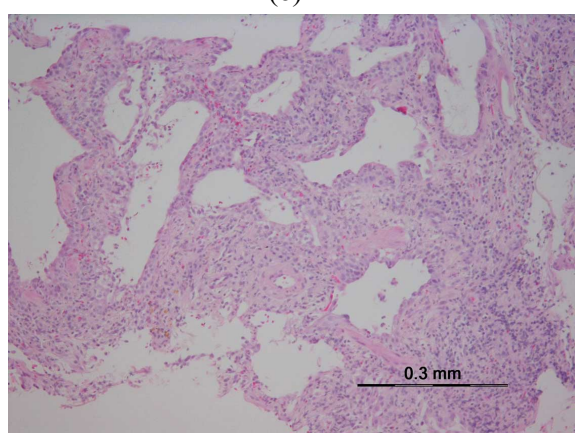

(d)

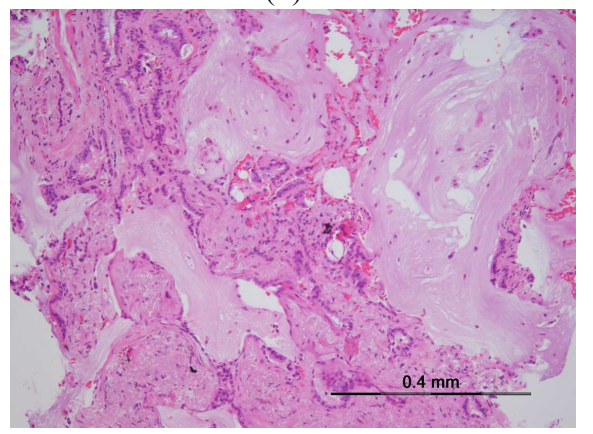

Fig. (1). Characteristic histological patterns (Hematoxylin \& Eosin staining in 100-200x magnification) in drug-induced interstitial lung disease, for example. (a) Hypersensitivity Pneumonia (a moderate chronic interstitial inflammation-the infiltrate consists of lymphocytes, plasma cells and multinucleated giant cells). (b) Organizing Pneumonia (airspaces are partially occupied by fibroblastic proliferations with collagen production). (c) Non specific interstitial Pneumonia (a homogeneous fibrotic broadening of the alveolar septa is seen with mild chronic inflammation, accompanied by hyperplasia of type II pneumocytes. (d) Usual interstitial Pneumonia (lung parenchyma with so-called honeycombing pattern-the lesion is characterized by cystic spaceswhich are surrounded by a dense fibrotic tissue. 
Table 3. Drugs with Toxic Pulmonary Effects

\begin{tabular}{|l|l|}
\hline \multicolumn{1}{|c|}{ Condition } & \multicolumn{1}{|c|}{ Drug } \\
\hline \hline Interstital pneumonia & $\begin{array}{l}\text { Adalimumab, Amphotericin B, Amiodarone, Azathioprine, Bleomycin, Busulfan, Chlorambucil, } \\
\text { Cyclophosphamide, Etanercept, Flecainide, Gold, Interferon alfa, Interferon beta, Infliximab, Melphalan, } \\
\text { Methadone, Methotrexate, Mexiletine, Nitrofurantoin, Paclitaxel, Penicillamine, Phenytoin, Rituximab, } \\
\text { Sirolimus, Statins, Sulfasalazine, }\end{array}$ \\
\hline Hypersensitivity pneumonitis & Azathioprine, 6-Mercaptopurine, beta-blockers, Busulfan, Fluoxetine, Nitrofurantoin, Procarbazine \\
\hline Bronchiolitis obliterans organizing pneumonia & $\begin{array}{l}\text { Amiodarone, Amphotericin, Bleomycin, Carbamazepine, Cocaine, Cyclophosphamide, Interferon alfa, } \\
\text { Interferon beta, Methotrexate, Penicillamine, Phenytoin, Sulfasalazine, Tetracyclines }\end{array}$ \\
\hline Granulomatous pneumonitis & Cocaine, Cromolyn sodium, Fluoxetine, Methotrexate, Nitrofurantoin, Pentozocine, Procarbazine \\
\hline
\end{tabular}

interstitium. Nodular organizing pneumonia is typically seen in patients exposed to bleomycin, in the form of roundshaped foci that localize mainly in lung bases, but may abut the pleura and simulate metastatic nodules (Fig. 1b).

\section{Interstitial Pneumonia}

Interstitial pneumonia, the most common manifestation of DILD, is an inflammation of the lung interstitium, such as alveolar septa. Drug-induced NSIP is a relatively common pulmonary reaction to drugs (Table 3 ). The typical pattern of NSIP is a uniform process affecting the lung homogenously suggesting a temporally uniform process. Lymphocytes, lymphoid aggregates and plasma cells infiltrate the alveolar septa and the peribronchial spaces. Interstitial fibrosis is also found [76 -78]. In drug-induced NSIP (Fig. 1c), interstitial inflammation is typically more homogeneous and more cellular than that seen in cases of usual interstitial pneumonia (UIP) (Fig. 1d). A wide array of drugs can cause interstitial pneumonia [2]. For example, the most common type of DILD due to anti-TNF agents is diffuse interstitial lung disease or pulmonary fibrosis, reported at 0.5 to $0.6 \%$ of at-risk patients $[41,79,80]$.

\section{Bronchiolitis Obliterans Organizing Pneumonia}

BOOP shows organized polypoid granulation inflammatory tissue in the distal bronchiole airways, respiratory bronchioles, alveolar ducts, and alveoli (Table 3). There is no disruption of the lung architecture and there is no traction bronchiectasis and no histological honeycombing [81].

\section{Granulomatous Pneumonitis}

Some drugs are capable of producing a granulomatous inflammation without necrosis (Table 3). These agents can induce a granulomatous pneumonitis with or without the bronchiolitis and interstitial inflammation seen in HP [82].

\section{DIAGNOSTIC PROCEDURES}

The diagnosis of DILD is mainly one of exclusion and requires the meticulous ruling out of all other possible causes. Unfortunately, therapeutic agents that cause DILD are often used to treat disease that can result in diffuse interstitial lung changes. It is, therefore, frequently difficult to determine if pulmonary abnormalities are related to the underlying disease or due to the medication. Discontinuance of the offending agent is often followed by spontaneous improvement, whereas failure to appreciate the causal relationship between the drug and the pulmonary disease can lead to irreversible lung injury or death. The diagnosis of DILD is usually based on several criteria [83-85]: (1) A history of drug exposure with correct identification of the drug, its dose, and its duration of administration. (2) Clinical, imaging and histopathological patterns which are consistent with earlier observations with the same drug. (3) Exclusion of other lung disease. (4) Improvement following discontinuation of the suspected drug and (5) Recurrence of symptoms on rechallenge, but rechallenge can be hazardous.

Numerous methods for causality assessment of ADR have been published and are falling into three categories: expert judgements, algorithms and probabilistic methods [86]. Expert judgements are individual assessments based on previous knowledge and experience in the field using no standardized tool to arrive at conclusions regarding causality. Algorithms are sets of specific questions with associated scores for calculating the likelihood of a cause-effect relationship. Bayesian approaches use specific findings in a case to transform the prior estimate of probability into a posterior estimate of probability of drug causation. Unfortunately, there is still no method universally accepted for causality assessment of ADR.

An objective assessment of the patient's baseline pulmonary status, as well as treatment history, is crucial to differentiate drug-induced pathology from the primary process. Diagnostic work-up should include a careful examination, laboratory studies, chest radiography and/or high-resolution CT (HRCT) of the chest, pulmonary function testing and-if necessary-invasive procedures such as bronchoscopy. Other non-invasive studies that may be helpful include an echocardiogram, sputum Gram stain and culture, and immunologic studies excluding vasculitis and connective tissue diseases.

The comparison of the observed case and previous cases is a very important part of diagnostic procedure. The spontaneous reporting system in pharmacovigilance is a process of collecting, assessing, presenting and interpreting suspected ADR. Causality assessment in pharmacovigilance may involve making decision based on the information on the relationship between a drug exposure and suspected ADR from a single adverse event or aggregated date. Standardized causality assessment is now a routine procedure at pharmacovigilance centers around the world [87]. 


\section{Clinical Signs/Symptoms and Physical Examination}

Many of the DILD have similar clinical features and are not easily distinguished on examination. Time to onset is from a few days to years and is unpredictable. The onset of the disease may be progressive over a few weeks, with isolated fever followed by the insidious development of respiratory symptoms, or the onset may be abrupt. Acute pneumonitis secondary to drug therapy can present with acute breathlessness occurring over several hours or days. Fever, rash, wheeze and peripheral eosinophilia are features of these reactions. The chronic form of the disease manifests as decreased exercise tolerance mainly as a result of progressive dyspnea. In the case of pulmonary hemorrhage, patients usually present with hemoptysis, dyspnea, hypoxemia, and acute anemia. It is also important to be aware that DILD may be enhanced by other factors such as age, impaired renal function, smoking and radiation therapy [88].

The physical findings of DILD are nonspecific. Physical examination reveals crackles on respiratory examination and may include digital clubbing. Signs of pulmonary hypertension with right ventricular dysfunction, such as lower-extremity edema or jugular venous distention, can occur late in the course and are not helpful in diagnosing a specific DILD.

\section{Laboratory Studies}

In general, laboratory analyses do not help in establishing the diagnosis. The white blood count may show increased eosinophils in cases of drug-induced pulmonary eosinophilia. However, the absence of peripheral eosinophilia does not exclude a diagnosis of drug-induced eosinophilic pneumonia. In the case of HP, peripheral eosinophilia may be present. Testing for antinuclear antibodies, anti-cytoplasmatic antibodies and anti-glomerular basal membrane autoantibodies is essential to classify druginduced DAH (autoimmune vs non-autoimmune), and to separate drug-induced DAH from DAH related to a naturally occurring systemic illness [89], for example the determination of anti-cytoplasmatic antibodies may be appropriate for the drug PTU.

\section{Radiology}

Generally, it is difficult to infer the histopathological background of the drug reaction from the pattern on imaging [90-98]. HRCT is more sensitive than chest radiography for defining the radiographic abnormalities, but is rarely specific for a drug etiology [99, 100]. One study reported abnormal findings on HRCT in all patients and abnormal findings on radiography in $74 \%$ [96]. On the other hand, the changes of classic radiation pneumonitis develop 1-2 months after the beginning of treatment in the form of a discrete haze, illdefined patchy nodules or an area of condensation with volume loss. The changes predominate in the irradiated area [101].

The pattern of involvement did not always correspond to the underlying histological findings and include NSIP, UIP, HP or BOOP (Fig. 2). Conclusively, HRCT is of limited value in determining the prognosis, and should be only performed in accordance with established guidelines and interpreted by a radiologist experienced in the evaluation of diffuse lung disease [102, 103]. (a)

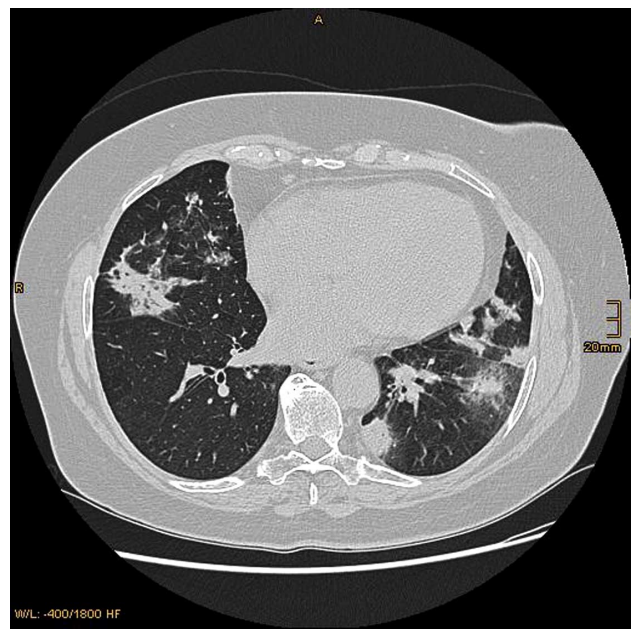

(b)

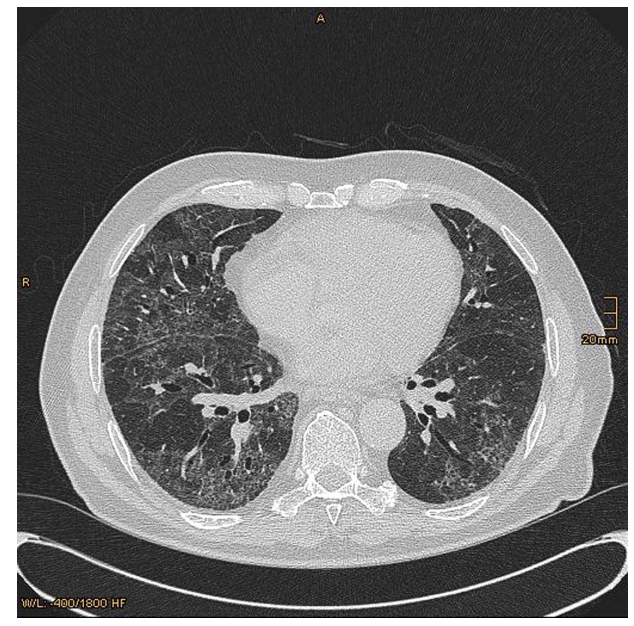

(c)

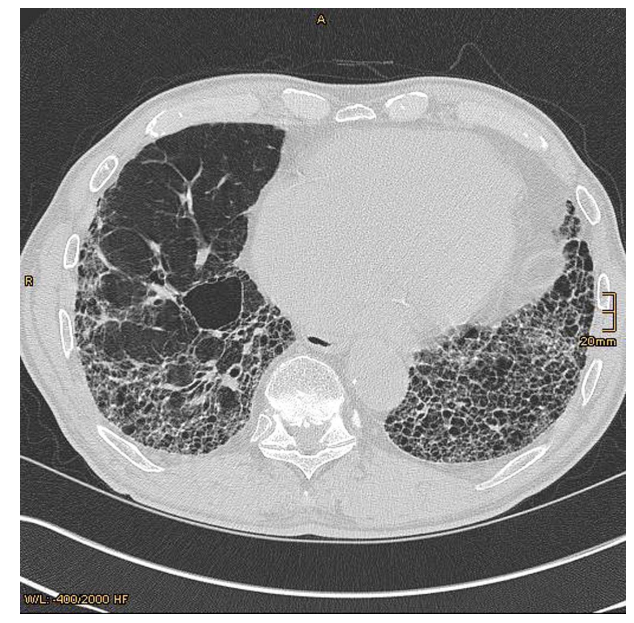

Fig. (2). Radiologic examples of drug induced interstitial lung disease, for example. (a) Bronchiolitis obliterans organizing Pneumonia. (b) Non specific interstitial Pneumonia. (c) Usual interstitial Pneumonia.

\section{Non-Specific Interstitial Pneumonia}

HRCT most often shows patchy ground-glass opacities or consolidation and irregular reticular opacities [96]. Typically 
these have a peripheral and basilar predominance. NSIP is mostly caused by amiodarone, methotrexate, and carmustine [100]. Lung parenchymal opacities in amiodarone have increased attenuation and this finding is suggestive, but it is not pathognomic of amiodarone-induced pulmonary toxicity (Fig. 2b).

\section{Usual Interstitial Pneumonia}

Cytotoxic chemotherapeutic agents, such as bleomycin and methotrexate, are the most common cause of UIP. Early high-resolution CT scans may show only scattered or diffuse areas of ground-glass opacity. Later, findings of fibrosis (traction bronchiectasis, honeycombing) predominate in a basal distribution. The predominance feature on HRCT is fibrosis that leads to subpleural cystic airspaces with thick walls that is known as "honeycombing" (Fig. 2c). Ground glass abnormalities, increased attenuation of the lung tissue without distortion of the underlying blood vessels or bronchi, are absent or minimal in classic UIP.

\section{Hypersensitivity Pneumonia}

Several drugs, for instance cyclophosphamide, sulfonamides or nonsteroidal anti-inflammatory drugs, can result in a hypersensitivity reaction in the lungs. Bilateral patchy ground-glass opacities with upper lobe-predominant centrilobular ill-defined nodules are the most common HRCT finding. In chronic disease, features of NSIP or UIP with fibrosis may predominate.

\section{Bronchiolitis Obliterans Organizing Pneumonia}

HRCT findings of BOOP show patchy ground-glass opacities with airbronchogramms and usually located peripherally (Fig. 2a). They may be triangular-shaped with the base of the triangle on the pleural surface. There are more than 35 drugs that cause BOOP including minocycline, bleomycin, amiodarone, phytoin, and the interferons [81].

\section{Pulmonary Function Testings}

The above-mentioned pathologic abnormalities can lead to profound impairment in lung physiology. Pulmonary function testing (PFT) can vary from an obstructive ventilatory defect due to bronchospasm or bronchiolitis obliterans to restrictive physiology with diffusion impairment secondary to pulmonary fibrosis. Most drugs cause a restrictive lung disease pattern with decreased total lung capacity (TLC), residual volume (RV), forced vital capacity (FVC), and diffusing capacity (DLCO) reflecting a pathologic disturbance of the alveolar-capillary interface [104]. The forced expiratory volume in one second $\left(\mathrm{FEV}_{1}\right)$ to $\mathrm{FVC}$ ratio $\left(\mathrm{FEV}_{1} / \mathrm{FVC}\right.$ ratio) may be normal or increased. However, drugs that cause bronchiolitis obliterans may cause an obstructive ventilatory defect (reduced $\mathrm{FEV}_{1} / \mathrm{FVC}$ ratio and $\mathrm{FEV}_{1}$, increased RV and RV/TLC ratio).

Gas exchange is impaired due to ventilation-perfusion mismatching and decreased diffusion across the abnormal interstitium. Arterial blood gas analysis may reveal hypoxemia at rest. Patients with DILD should have also arterial oxygen saturation determined during exertion, because many patients with only mild disease desaturate with exertion despite normal saturation at rest.

\section{Bronchoscopy}

Bronchoscopy with bronchoalveolar lavage (BAL) is a minimally invasive, well-tolerated clinical tool that can be useful in evaluating patients who have diffuse infiltrative lung disease. Although it is infrequently diagnostic by itself, when combined with clinical data and radiographic imaging, a likely diagnosis often can be reached [105]. The results of BAL cell differentials with a lymphocytic $(\geq 15 \%)$, a neutrophilic $(\geq 3 \%)$, an eosinophilic $(\geq 2 \%)$, or a mixed cellular pattern can be used as an adjunct to diagnosis [106, 107]. BAL findings in drug-induced HP include lymphocytosis, a low CD4 to CD8 ratio, and occasionally, an increase in neutrophils. Eosinophilic pneumonia is typified by an elevated eosinophilic count in the BAL fluid [108]. Cytotoxic pneumonitis due to chemotherapeutic agents typically has a neutrophil predominance. BAL findings in drug-induced BOOP show increased lymphocytes. Exceptionally some drugs cause characteristic changes in the $\mathrm{BAL}$, for example amiodarone with changes in the alveolar macrophage population, which shows foamy intracytoplasmic alterations and corresponds to a form of phospholipidosis [49]. Unfortunately, these findings indicate medication exposure and do not necessarily establish drug toxicity. Table 4 describe typical BAL findings in DILD due to several agents.

Table 4. Bronchoalveolar Lavage (BAL) Cell Differentials as an Adjunct to Diagnosis (Adapted from 108 and 109)

\begin{tabular}{|c|c|}
\hline Typical BAL Findings & Examples \\
\hline \multirow{5}{*}{ Neutrophilic } & Idiopathic pulmonary fibrosis \\
\hline & Acute respiratory distress syndrome \\
\hline & Connective tissue disorders \\
\hline & Asbestosis \\
\hline & Wegener`s granulomatosis \\
\hline \multirow{4}{*}{ Eosinophilic } & Allergic bronchopulmonary aspergillosis \\
\hline & Churg Strauss syndrome \\
\hline & Eosinophilic pneumonia \\
\hline & Idiopathic hypereosinophilic syndrome \\
\hline \multirow{5}{*}{ Lymphocytic } & Sarcoidosis \\
\hline & Berylliosis \\
\hline & Hypersensitivity pneumonitis \\
\hline & Silicosis \\
\hline & Crohn disease \\
\hline \multirow{3}{*}{ Mixed cellularity } & BOOP \\
\hline & Connective-tissue disease \\
\hline & Nonspecific interstitial pneumonia \\
\hline
\end{tabular}

Although open lung biopsies are not pathognomonic for drug toxicity and correlation with clinical, laboratory, and radiologic data is required, they can be a tool in the evaluation of suspected DILD by helping to exclude 
underlying disease or infection and documenting the pattern of lung injury. The latter information is helpful in making the diagnosis of drug toxicity as well as guiding the optimal management of the patient. Moreover, there are exceptions with characteristic histological findings, such free foamy macrophages supporting the diagnosis of amiodarone pulmonary toxicity. Although a surgical lung biopsy might allow the pathologist to give a more complete description of the pulmonary process, a minimally-invasive procedure, such as BAL or bronchoscopic biopsy, can contribute valuable data [2, 109-111]. Mostly, the amount of lung tissue obtained by transbronchial biopsy is very small and their findings, particularly those that are themselves non-specific, should not be anyway considered diagnostic. Cockerill and colleagues [112] found that $20 \%$ of patients with diffuse infiltrates who underwent surgical lung biopsy had pathologic findings that could be attributed to a drug reaction. In the study of Romagnoli et al., transbronchial lung biopsy samples were considered adequate and diagnostic, and confirmed the diagnosis of DILD in $76 \%$ of the cases [111]. Furthermore, correlations of the histology with HRCT by pathologists and radiologists have contributed to our knowledge [93, 98].

\section{TREATMENT}

The primary goal of treatment is to suppress the inflammatory response and prevent the deposition of fibrotic tissue. The management strategy depends on the severity of the disease. In the first step, the medication should be withdrawn and the treatment of DILD consists appropriately managing of pulmonary symptoms, after other possibilities are eliminated and DILD is highly suspected. Ideally, symptoms should remit and management includes supportive care. Acute episodes of drug-induced pulmonary disease usually disappear 24-48 hours after the drug has been discontinued, but chronic syndromes may take longer to resolve. Because hypoxemia is common in DILD, supplemental oxygen therapy is often prescribed.

Secondly, if the cytotoxic drug-induced disease is severe or appears to progress despite elimination of further drug exposure, an empirical course of glucocorticoids is advisable. In certain DILD such as BOOP, eosinophilc pneumonia, and HP, there is evidence that corticosteroids may hasten resolution of symptoms. Drug induced acute eosinophilic pneumonia generally responds to drug withdrawal and corticosteroid therapy, but tends to relapse if the patient is rechallenged with the drug, especially in the context of early corticosteroid withdrawal. Thirdly, in other entities such as pulmonary fibrosis, corticosteroids have no role, and the disease may be progressive even after withdrawal of the offending agent. If a patient is rechallenged with the drug, symptoms may or may not recur. This is a decision that has to be carefully weighed depending on the severity of the drug-related pulmonary toxicity and the morbidity associated with not treating the underlying disease. If alternative agents are available, they should be used.

Because many patients with DILD are treated with immunosuppressive medications and are at some modest increased risk for the development of infections, patients with DILD should receive a pneumococcal vaccine and a yearly influenza virus vaccine. Furthermore, tuberculosis may be associated with anti-tumor necrosis factor monoclonal antibody therapy. The increased risk with early anti-TNF treatment and the absence of correct chemoprophylactic treatment favor the reactivation of latent tuberculosis [113]. Greater awareness is necessary of patients with risk factors, particularly ethnicity, to facilitate more appropriate targeting of chemoprophylaxis [114].

\section{PROGNOSIS}

Prognosis of acute DILD may be satisfactory, if the diagnosis is performed early. Therefore, a full recovery could be achieved. On the other hand, failure to recognize a drug-mediated lung disease can lead to significant morbidity and mortality. The prognosis is variable and depends on the specific drug and underlying clinical, physiologic, and pathologic severity of the lung disease. Typical complications of DILD are pulmonary fibrosis and respiratory failure requiring mechanical ventilation. Unfortunately, if the initiating injury or abnormal repair from injury is not halted, progressive tissue damage can lead to worsening physiologic impairment and even death.

The prognosis does not seen to be linked to the severity of the initial clinical picture or to the nature of the underlying neoplastic disorder, but to the degree of the pulmonary fibrosis [114]. The mortality in acute amiodarone pulmonary toxicity approaches 40-50\%, despite drug withdrawal and corticosteroid therapy. Overall, the mortality in amiodarone pulmonary toxicity is less than $10 \%$ in ambulatory patients, but it is higher (20-33\%) in patients who are diagnosed later or who require admission to the hospital for this condition $[101,115]$. In methotrexate lung, a mortality rate of $15 \%$ in one series underlines the need for careful management of this condition [116].

\section{CONCLUSIONS}

Now there are over 450 drugs recognizing as being implicated in interstitial lung disease. At present, there is no consensus for a definite diagnostic workup approach in patients with a suspicion of diffuse DILD. Clinical and radiographic features of diffuse DILD are often difficult to distinguish from other causes of diffuse lung disease (e.g. infections, lung involvement of an underlying malignancy, pulmonary edema, connective tissue disease), and there are no signs, symptoms, laboratory or radiologic data that could be considered as pathognomonic.

Therefore, it is important for physicians to be familiar with iatrogenic diseases for which their patients are at risk. Despite better appreciation of DILD, patients may still die from acute or chronic DILD [117, 118]. DILD is preventable to a certain extent, and sources of improvement include better information to patients, avoidance of certain drugs in allergic patients, and earlier diagnosis [118, 119]. Serial lung function testing are still considered useful in patients on bleomycin, less so in those on amiodarone or methotrexate [120,121].

It is evident that DILD represent a very heterogenous group of lung disease representing one of the fundamental injurious responses of the lung. However, the pathogenetic mechanism underlying DILD are still mostly unknown, especially in humans. Most experimental studies have been 
conducted with animals exposed to drugs, and these reactions cannot be directly extrapolated to human lung. Another limitation for studying DILD in human lung is the different occurrence of those diseases. It is very difficult to gather large numbers of patients/samples without international collaboration involving several specialities [74]. A systematic collection of human lung tissue and cell material would significantly improve the clinicalradiological-histopathological interpretation especially if combined with experimental in vitro studies in cells established from human lung.

With an increasing number of therapeutic drugs available for use, the list of drugs that is responsible for severe pulmonary disease also grows. Genetic polymorphism of drug-metabolizing enzymes, particularly of the cytochrome P450 superfamily of enzymes, influences individual drug efficacy and safety through the alteration of pharmacokinetics and disposition of drugs. In the future genotyping should be considered to identify patients who are at high risk of severe toxic responses, in order to guide appropriate individual dosage. Both clinical and genetic risk stratification may lead to more accurate prevention of DILD in the future.

\section{ACKNOWLEDGEMENT}

Declared none.

\section{AUTHOR CONTRIBUTIONS/FINANCIAL \& COMPE- TING INTEREST DISCLOSURE}

All authors participated in interpreting the data and revising the manuscript for important intellectual content. All authors approved the final version of the manuscript. The authors have no relevant affiliations or financial conflict with the subject matter or materials discussed in the manuscript. No writing assistance was utilized in the production of this manuscript.

\section{ABREVIATIONS}

$$
\begin{aligned}
& \text { ADR }=\text { Adverse drug reactions } \\
& \text { ARDS }=\text { Acute respiratory distress syndrome } \\
& \text { BAL }=\text { Broncho-alveolar lavage } \\
& \text { BOOP }=\text { Bronchiolitis obliterans organizing pneumonia } \\
& \text { OP }=\text { Organizing pneumonia } \\
& \text { DILD }=\text { Drug induced interstitial lung disease } \\
& \text { DLCO }=\text { Diffusing capacity } \\
& \text { FEV1 }=\text { Forced expiratory volumen in one second } \\
& \text { FVC }=\text { Forced vital capacity } \\
& \text { HP }=\text { Hypersensitivity pneumonitis } \\
& \text { HRCT }=\text { High resolution computed tomography } \\
& \text { ILD }=\text { Interstitial lung disease } \\
& \text { INF }=\text { Interferon } \\
& \text { NSAIDs }=\text { Nonsteroidal anti-inflammatory drugs } \\
& \text { NSIP }=\text { Non-specific interstitial pneumonia } \\
& \text { PFT }=\text { Pulmonary function testing }
\end{aligned}
$$
$\mathrm{RV} \quad=$ Residual volume
TLC $=$ Total lung capacity
$\mathrm{TNF}=$ Tumor necrosis factor
UIP $=$ Usual interstitial pneumonia

\section{REFERENCES}

[1] Camus P, Rosenow E. Latrogenic lung disease. Clin Chest Med 2004; 25: xiii-xix.

[2] Flieder D, Travis W. Pathologic characteristics of drug-induced lung disease. Clin Chest Med 2004; 25: 37-45.

[3] Nemery B, Bast A, Behr J, et al. Interstitial lung disease induced by exogenous agents: factors governing susceptibility. Eur Respir J 2001; 18: 30S -42S.

[4] Spear B, Heath-Chiozzi M, Huff J. Clinical application of pharmacogenetics. Trends Mol Med 2002; 7: 201-4.

[5] Lazarou J, Pomeranz B, Corey P. Incidence of adverse drug reactions in hospitalized patients: a meta-analysis of prospective studies. JAMA 1998; 279: 1200-5

[6] Beijer H, de Blaey C. Hospitalisations caused by adverse drug reactions (ADR): a meta-analysis of observational studies. Pharm World Sci 2002; 24: 46-54.

[7] Hitchen L. Adverse drug reactions result in 250000 UK admissions a year. BMJ 2006; 332: 1109.

[8] Wester K, Jonnson A, Sigset O, et al. Incidence of fatal adverse drug reactions: a population based study. Br J Clin Pharmacol 2008; 65: 573-9.

[9] Davies E, Green C, Talor S, et al. Adverse drug reactions in hospital in-patients. A prospective analysis of 3695 patientepisodes. PLoS One 2009; 4: e4439.

[10] Wu T, Jen M, Bottle A, et al. Ten-year trends in hospital admissions for adverse drug reactions in England 1999-2009. J R Soc Med 2010; 103: 239- 50.

[11] Limper A, Rosenow E. Drug-induced interstitial lung disease. Curr Opin Pulm Med 1996; 2: 396-404.

[12] Coultas D, Zumwalt R, Black W, et al. The epidemiology of interstitial lung diseases. Am J Respir Crit Care Med 1994; 150: 967-72.

[13] Thomeer M, Costabel U, Rizzato G, et al. Comparison of registries of interstitial lung diseases in three European countries. Eur Respir J 2001; 18: 114s-8s.

[14] Pneumotox online: the drug-induced lung diseases. Available from: www.pneumotox.com [Accessed February 2012].

[15] Simpson A, Paul J, Graham J, Kaye S. Fatal bleomycin pulmonary toxicity in the west of Scotland 1991-95: a review of patients with germ cell tumours. Br J Cancer 1998; 78: 1061-6.

[16] Mendez J, Nardous H, Hartman T, et al. Chronic nitrofurantoininduced lung disease. Mayo Clin Proc 2005; 80: 1298-302.

[17] Kudoh S, Kato H, Nishiwaki Y, et al. Japan Thoracic Radiology Group Interstitial lung disease in Japanese patients with lung cancer: a cohort and nested case-control study. Am J Respir Crit Care Med 2008; 177: 1348-57.

[18] Miyakoshi S, Kami M, Yuji K, et al. Severe pulmonary complications in Japanese patients after bortezomib treatment for refractory multiple myeloma. Blood 2006; 107: 3492-4.

[19] Ohri A, Arena F. Severe pulmonary complications in AfricanAmerican patient after bortezomib therapy. Am J Ther 2006; 13 553-5.

[20] Suissa S, Hudson M, Ernst P. Leflunomide use and the risk of interstitial lung disease in rheumatoid arthritis. Arthritis Rheum 2006; 54: 1435-9.

[21] Miwa Y, Isozaki T, Wakabayashi K, et al. Tacrolimus-induced lung injury in a rheumatoid arthritis patient with interstitial pneumonitis. Mod Rheumatol 2008; 18: 208-11.

[22] Empey P. Genetic predisposition to adverse drug reactions in the intensice care unit. Crit Care Med 2010; 38: S106-16.

[23] Ingelman-Sundberg M, Sim S, Gomez A, et al. Influence of cytochrome P450 polymorphisms on drug therapies: pharmacogenetic, pharmacoepigenetic and clinical aspects. Pharmacol Ther 2007; 116: 496-526.

[24] Wijnen P, Drent M, Nelemans P, et al. Role of cytochrome P450 polymorphisms in the development of pulmonary drug toxicity: a case-control study in the Netherlands. Drug Saf 2008; 31: 1125-34. 
[25] Cavallari L, Limdi N. Warfarin pharmacogenomics. Curr Opin Mol Ther 2009; 11: 243-51.

[26] Limdi N, McGwin G, Goldstein J, et al. Influence of CYP2C9 and VKORC1 $1173 \mathrm{C} / \mathrm{T}$ genotype on the risk of hemorrhagic complications in African-American and European-American patients on warfarin. Clin Pharmacol Ther 2008; 83: 312-21.

[27] Vorperian V, Havighurst T, Millrer S, January C. Adverse effects of low dose amiodarone: A meta-analysis. J Am Coll Cardiol 1997; 30: 791-8.

[28] Yamada Y, Shiga T, Matsuda N, et al. Incidence and predictors of pulmonary toxicity in Japanese patients receiving low-dose amiodarone. Circ J 2007; 71: 1610-6.

[29] Aronin P, Mahaley M, Rudnick S, et al. Prediction of BCNU pulmonary toxicity in patients with malignant gliomas: an assessment of risk factors. N Engl J Med 1980; 303: 183 -8.

[30] Stemmer S, Cagnoni P, Shpall E, et al. High-dose paclitaxel, cyclophosphamide, and cisplastin with autologous hematopoietic progenitor-cell support: a phase I trial. J Clin Oncol 1996; 14: 1463-72.

[31] Parish J, Muhm J, Leslie K. Upper lobe pulmonary fibrosis associated with high-dose chemotherapy containing BCNU for bone marrow transplantation. Mayo Clin Proc 2003; 78: 630-4.

[32] Bast A, Weseler A, Haenen G, den Hartog G. Oxidative stress and antioxidants in interstitial lung disease. Curr Opin Pulm Med 2010, 16: 516-20.

[33] Crimi E, Sica V, Williams-Ignarro S, et al. The role of oxidative stress in adult critical care. Free Radic Biol Med 2006; 40: 398406.

[34] Denman J, Gilbar P, Abdi E. Hypersensitivity reaction (HSR) to docetaxel after a previous HSR to paclitaxel. J Oncol 2002; 20: 2760-1.

[35] Oya N, Sasai K, Tachiiri S, et al. Influence of radiation dose rate and lung dose on interstitial pneumonitis after fractionated total body irradiation: acute parotitis may predict interstitial pneumonitis. Int J Hematol 2006; 83: 86-91

[36] Barrera P, Laan R, van Riel P, et al. Methotrexate-related pulmonary complications in rheumatoid arthritis. Ann Rheum Dis 1994; 53: 434-9.

[37] Alarcón G, Kremer J, Macaluso M, et al. Risk factors for methotrexate-induced lung injury in patients with rheumatoid arthritis. Ann Intern Med 1997; 127: 356-64.

[38] Keysser G. Methotrexate toxicity: Myths and facts. Z Rheumatol 2011; 70: 108-13.

[39] Sathi N, Chikura B, Kaushik V, et al. How common is methotrexate pneumonitis? A large prospective study investigates. Clin Rheumatol 2012; 31: 79-83.

[40] Ju J, Kim S, Lee J, et al. Risk of interstitial lung disease associated with leflunomide treatment in Korean patients with rheumatoid arthritis. Arthritis Rheum 2007; 56: 2094-6.

[41] Koike T, Harigai M, Inokuma S, et al. Postmarketing surveillance of the safety and effectiveness of etanercept in Japan. J Rheumatol 2009; 36: 898-906.

[42] Sawada $\mathrm{T}$, Inokuma $\mathrm{S}$, Sato $\mathrm{T}$, et al. Leflunomide-induced interstitial lung disease: prevalence and risk factors in Japanese patients with rheumatoid arthritis. Rheumatology 2009; 48: 106972.

[43] Danson S, Blackhall F, Hulse P, Ranson M. Interstitial lung disease in lung cancer: separating disease progression from treatment effects. Drug Saf 2005; 28: 103-13.

[44] Copper J. Drug-induced pulmonary disease. Adv Intern Med 1997; 42: 231-68.

[45] Ohnishi K, Sakai F, Kudoh S, Ohno R. Twenty-seven cases of drug-induced interstitial lung disease associated with imatinib mesylate. Leukemia 2006; 20: 1162-4.

[46] Roig J, Domingo C, Gea E. Pulmonary Toxicity Caused by Cytotoxic Drugs. Clin Pulm Med 2006; 13: 53-62.

[47] Jules-Elysee K, White D. Bleomycin induced pulmonary toxicity. Clin Chest Med 1990; 11: 1-20.

[48] Aronchik J, Gefter W. Drug-induced pulmonary disorders. Semin Roentgenol 1995; 30: 18-34.

[49] Schwaiblmair M, Berghaus T, Haeckel T, et al. Amiodaroneinduced pulmonary toxicity: an underrecognized and severe adverse effect? Clin Res Cardiol 2010; 99: 693-700.

[50] Fernández A, Karas R, Alsheikh-Ali A, Thompson P. Statins and interstitial lung disease: a systematic review of the literature and of food and drug administration adverse event reports. Chest 2008; 134: 824-30.

[51] Zitnik R, Cooper J. Pulmonary disease due to antirheumatic agents. Clin Chest Med 1990; 11: 139-50.

[52] Sharma A, Provenzale D, McKusick A, et al. Interstitial pneumonitis after low-dose methotrexate therapy in primary biliary cirrhosis. Gastroenterology 1994; 107: 266-70.

[53] Ramos-Casals M, Perez-Alvarez R, Perez-de-lis M, et al. Pulmonary disorders induced by monoclonal antibodies in patients with rheumatologic autoimmune diseases. Am J Med 2011; 124 : 386-94.

[54] Bhullar S, Lele S, Kraman. Severe nitrofurantoin lung disease resolving without the use of steroids. J Postgrad Med 2007; 53: 111-3.

[55] Giezen T, Mantel-Teeuwisse A, Straus S, et al. Safety-related regulatory actions for biologicals approved in the United States and the European Union. JAMA 2008; 300: 1887-96.

[56] Walsh G. Biopharmaceutical benchmarks 2006. Nat Biotechnol 2006; 24: 769-76.

[57] Peerzada M, Spiro T, Daw H. Pulmonary toxicities of biologics: a review. Anti-Cancer Drugs 2010; 21: 131-9.

[58] Zayen A, Rais H, Ouarda M, et al. Rituximab-Induced Interstitial Lung Disease: Case Report and Literature Review. Pharmacology 2011; 87: 318-20.

[59] Domingo C, Roig J. Neglected Respiratory Toxicity Caused by Cancer Therapy. Open Respir Med J 2007; 1: 1-6.

[60] Özkan M, Dweik R, Ahmad M. D. Drug induced lung disease. Cleve Clin J Med 2001; 68: 782-95.

[61] Belmonte $Y$, de Fàbregues $O$, Martin $M$, Domingo $C$. Pleuropulmonary Toxicity of Another Anti-Parkinson's Drug: Cabergoline. Open Resp Med J 2009; 3: 90-3.

[62] Shimabukuro D, Sawa T, Gropper M. Injury and repair in lung and airways. Crit Care Med 2003; 31: S524-S31.

[63] Delaunois L. Mechanisms in pulmonary toxicology. Clin Chest Med 2004; 1-14.

[64] Foucher P, Biour M, Blayac J, et al. Drugs that may injure the respiratory system. Eur Respir J 1997; 10: 265-79.

[65] Weinshilboum R. Inheritance and drug response. N Engl J Med 2003; 348: 529-37.

[66] Higenbottam T, Kuwano K, Nemery B, et al. Understanding the mechanism of drug-associated interstitial lung disease. Br J Cancer 2004; 91: S31-7.

[67] Ryrfeldt A. Drug-induced inflammatory responses to the lung. Toxicol Lett 2000; 112-113: 171-6.

[68] Mishra A, Doyle N, Martin W. Bleomycin-mediated pulmonary toxicity. Am J Respir Cell Mol Biol 2000; 22: 543-9.

[69] Azambuja E, Fleck J, Batista R, Barreto M. Bleomycin lung toxicity: who are the patients with increased risk? Pulmo Pharmacol Ther 2005; 18: 363-6.

[70] Uhal B. Apoptosis in lung fibrosis and repair. Chest 2002; 122 : 293S-8S.

[71] Lock B, Eggert M, Cooper J. Infiltrative lung disease due to noncytotoxic agents. Clin Chest Med 2004; 25: 47-52.

[72] Katzenstein A, Fiorelli R. Nonspecific interstitial pneumonia/fibrosis. Histologic features and clinical significance. Am J Surg Pathol 1994; 18: 136-47.

[73] Fukuoka J, Leslie K. Chronic diffuse lung diseases. In : Leslie K and Wick M, Ed. Practical Pulmonary Pathology. Philadelphia: Churchill-Livingstone 2005; pp 181-258.

[74] Petri W. Sulfonamides, sulfamethoxazole, quinolones and agents for urinary tract infections. In: Hardman J, Linford L, Eds, The Pharmacological Basics of Therapeutics, $10^{\text {th }}$ ed. Chapt 44 . New York: Goodman and Gilman`s, 2001; p. 1185.

[75] Kaarteenaho R, Kinnula V. Diffuse alveolar damage: A common phenomenon in progressive interstitial lung disorders. Pulm Med $2011 ; 2011 ; 531302$.

[76] Ostoer A, Chilvers E, Somerville M, et al. Pulmonary complications of infliximab therapy with rheumatoid arthritis. J Rheumatol 2006; 33: 622-8.

[77] Huggett M, Armstrong R. Adalimumab-associated pulmonary fibrosis. Rheumatology 2006; 45: 1312-3.

[78] Takeuchi T, Tatsuki Y, Nogami Y, et al. Postmarketing surveillance of the safety profile of infliximab in 5000 Japanese patients with rheumatoid arthritis. Ann Rheum Dis 2008; 67: 18994. 
[79] Pietra G. Pathologic mechanisms of drug-induced lung disorders. J Thorac Imaging 1991; 6: 1-7.

[80] Lioté H, Lioté F, Séroussi B, et al. Rituximab-induced lung disease: a systematic literature review. Respir J 2010; 35: 681-7.

[81] Epler G. Bronchiolitis obliterans organizing pneumonia (BOOP), 25 years: a variety of causes, but what are the treatment options? Expert Rev Respir Med 2011; 5: 353-61.

[82] Kornacker M, Kraemer A, Leo E, Ho A. Occurrence of sarcoidosis subsequent to chemotherapy for non-Hodgkin`s lymphoma: report of two cases. Ann Hematol 2002; 81: 103-5.

[83] Camus P. Drug induced infiltrative lung diseases. In: Schwarz M, King T, Eds. Interstitial disease, $4^{\text {th }}$ ed. London: BC Decker 2003; pp. 485-534.

[84] Camus P, Bonniaud P, Fanton A, et al. Drug-induced and iatrogenic infiltrative lung disease. Clin Chest Med 2004; 25: 479519.

[85] Bradley B, Branley HM, Egan JJ, et al. Interstitial lung disease guideline: the British Thoracic Society in collaboration with the Thoracic Society of Australia and New Zealand and the Irish Thoracic Society. Thorax 2008; 63: v1-v58.

[86] Agbabiaka T, Savovic J, Ernst E. Methods for causality assessment of adverse drug reactions. Drug Safety 2008; 31: 21-37.

[87] Meyboum R, Hekster Y, Egberts A, et al. Causal or casual? the role of causality assessment in pharmacovigilance. Drug Saf 1997; 17: 374-89.

[88] Ganguli A, Pirmohamed M. Management of drug-induced interstitial lung disease. Prescriber 2006; 5: 41-6.

[89] Choi H, Merkel P, Walker A, Niles J. Drug-associated antineutrophil cytoplasmatic antibody-positive vasculitis: prevalence among patients with high titers of antimyeloperoxidase antibodies. Arthritis Rheum 2000; 43: 405-13.

[90] Elliot T, Lynch D, Newell J, et al. High-resolution computed tomography features of nonspecific interstitial pneumonia and usual interstitial pneumonia. J Comput Assist Tomogr 2005; 29: 339-45.

[91] Hunninghake G, Lynch D, Galvin J, et al. Radiologic findings are strongly associated with a pathologic diagnosis of usual interstitial pneumonia. Chest 2003; 124: 1215-23.

[92] Morikawa M, Demura Y, Mizuno S, et al. FDG positron emission tomography imaging of drug-induced pneumonitis. Ann Nucl Med 2008; 22: 335-8.

[93] Cleverley J, Screaton N, Hiorns M, et al. Flint J, Müller N. Druginduced lung disease: high-resolution $\mathrm{CT}$ and histological findings. Clin Radiol 2002; 57: 292-9.

[94] Erasmus J, McAdams H, Rossi S, et al. High-resolution CT of drug-induced lung disease. Radiol Clin North Am 2002; 40: 61-72.

[95] Ellis S, Cleverly J, Müller N. Drug-induced lung disease: highresolution CT findings. AJR Am J Roentgenol 2000; 175: 1019-24.

[96] Padley S, Adler B, Hansell D, Müller N. High-resolution computed tomography of drug-induced lung disease. Clin Radiol 1992; 46: 232-6.

[97] Rossi S, Erasmus J, McAdams H, et al. Pulmonary drug toxicity: radiologic and pathologic manifestations. Radiographics 2000; 20 : 1245-59.

[98] Akira M, Ishikawa H, Yamamoto S. Drug-induced pneumonitis: Thin-section CT findings in 60 patients. Radiology 2002; 224: 85260 .

[99] Lindell R, Hartman T. Chest imaging in iatrogenic respiratory disease. Clin Chest Med 2004; 25: 15-24.

[100] Nishiyama O, Kondoh Y, Taniguchi H, et al. Serial high resolution CT findings in nonspecific interstitial pneumonia/fibrosis. J Comput Assist Tomogr 2000; 24: 41-6.
[101] Camus P, Fanton A, Bonniaud P, et al. Interstitial lung disease induced by drugs and radiation. Respiration 2004; 71: 301-26.

[102] American Thoracic Society/European Respiratory Society International Multidisciplinary Consensus Classification of the Idiopathic Interstitial Pneumonias. Am J Respir Crit Care Med 2002; 165: 277-304.

[103] Cleverley J, Screaton N, Hiorns M, et al. Drug-induced lung disease: high-resolution $\mathrm{CT}$ and histological findings. Clin Radiol 2002; 57: 292-9.

[104] Chetta A, Marangio E, Olivieri D. Pulmonary function testing in interstitial lung diseases. Respiration 2004; 71: 209-13.

[105] Meyer K. The role of bronchoalveolar lavage in interstitial lung disease. Clin Chest Med 2004; 25: 637-49.

[106] Costabel U, Uzaslan E, Guzman J. Bronchoalveolar lavage in druginduced lung disease. Clin Chest Med 2004; 25: 25-35.

[107] Domingo C, Roig J, Klamburg P, et al. Lavado broncoalveolar en Medicina intensiva. Medicina Intensiva 1988; 12: 159-66.

[108] Janz D, O'Neil H, Ely E. Acute eosinophilic pneumonia: a case report and review of the literature. Crit Care Med 2009; 37: 1470-4.

[109] Camus P, Costabel U. Drug-induced respiratory disease in patients with haematological diseases. Semin Respir Crit Care Med 2005; 26: 458-81.

[110] Cho I, Mori S, Imamura F, et al. Methotrexate pneumonia lacking dyspnea and radiographic interstitial patterns during treatment for early rheumatoid arthritis: bronchoalveolar lavage and transbronchial lung biopsy in a differential diagnosis. Mod Rheumatol 2007; 17: 256-61.

[111] Romagnoli M, Bigliazzi C, Casoni G, et al. The role of transbronchial lung biopsy for the diagnosis of diffuse druginduced lung disease: a case series of 44 patients. Sarcoidosis Vasc Diffuse Lung Dis 2008; 25: 36-45.

[112] Cockerill F, Wilson W, Carpenter H, et al. Open lung biopsy in immunocompromised patients. Arch Intern Med 1985; 145: 1398404.

[113] Tubach F, Salmon D, Ravaud P, et al. Risk of tuberculosis is higher with anti-tumor necrosis factor monoclonal antibody therapy than with soluble tumor necrosis factor receptor therapy: the three-year prospective French Research Axed on Tolerance of Biotherapies registry. Arthritis Rheum 2009; 60: 1884-94.

[114] John H, Buckley C, Koh L, et al. Regional survey of tuberculosis risk assessment in rheumatology outpatients commencing antiTNF-alpha treatment in relation to British Thoracic Society. Clin Med 2009; 9: 225-30.

[115] Chabot F, Aymard B, Lesur O, et al. Drug-induced pulmonary diseases: diagnostic, therapeutic and prognostic aspects. Apropos of 10 personal case reports. Rev Mal Resp 1992; 9: 593-601.

[116] Kremer J, Alarcon G, Weinblatt M, et al. Clinical, laboratory, and histopathologic features of methotrexate-associated lung injury in patients with rheumatoid arthritis: a multicenter study with literature review. Arthritis Rheum 1997; 40: 1829-37.

[117] Pumphrey R. Lessons from management of anaphylaxis from a study of fatal reactions. Clin Exp Allergy 2000; 30: 1144-50.

[118] Kharabsheh S, Abendroth C, Kozak M. Fatal pulmonary toxicity occurring within two weeks of initiation of amiodarone. Am J Cardiol 2002; 89: 896-8.

[119] Kuehm S, Doyle M. Medication errors: 1977 to 1988. Experience in medical malpractice claims. N J Med 1990; 87: 27-34.

[120] Cottin V, Tébib J, Massonnet B, Souquet P, Bernard J. Pulmonary function in patients receiving longterm low-dose methotrexate. Chest 1996; 109: 933-8.

[121] Verweij J, van Zanten T, Souren T, Golding R, Pinedo H. Prospective study on the dose relationship of mitomycin C-induced interstitial pneumonitis. Cancer 1987; 60: 756-61. 